\title{
Correlation between structural, magnetic, and dielectric properties of manganese substituted cobalt ferrite
}

\author{
C V. Ramana \\ Y D. Kolekar \\ K K. Bharathi \\ B Sinha \\ Kartik Ghosh \\ Missouri State University
}

Follow this and additional works at: https://bearworks.missouristate.edu/articles-cnas

\section{Recommended Citation}

Ramana, C. V., Y. D. Kolekar, K. Kamala Bharathi, B. Sinha, and K. Ghosh. "Correlation between structural, magnetic, and dielectric properties of manganese substituted cobalt ferrite." Journal of applied physics 114, no. 18 (2013): 183907.

This article or document was made available through BearWorks, the institutional repository of Missouri State University. The work contained in it may be protected by copyright and require permission of the copyright holder for reuse or redistribution.

For more information, please contact BearWorks@library.missouristate.edu. 


\title{
Correlation between structural, magnetic, and dielectric properties of manganese substituted cobalt ferrite
}

\author{
C. V. Ramana, ${ }^{1, a)}$ Y. D. Kolekar, ${ }^{1}$ K. Kamala Bharathi, ${ }^{1}$ B. Sinha,${ }^{2}$ and K. Ghosh ${ }^{3}$ \\ ${ }^{1}$ Department of Mechanical Engineering, University of Texas at El Paso, El Paso, Texas 79968, USA \\ ${ }^{2}$ Functional Nanopowder Material Division, Korea Institute of Material Science, Changwon 642-831, \\ Gyeongnam, South Korea \\ ${ }^{3}$ Department of Physics, Astronomy and Materials Science, Missouri State University, Springfield, \\ Missouri 65897, USA
}

(Received 13 August 2013; accepted 14 October 2013; published online 12 November 2013)

\begin{abstract}
Manganese (Mn) substituted cobalt ferrites $\left(\mathrm{CoFe}_{2-\mathrm{x}} \mathrm{Mn}_{\mathrm{x}} \mathrm{O}_{4}\right.$, referred to CFMO) were synthesized and their structural, magnetic, and dielectric properties were evaluated. X-ray diffraction measurements coupled with Rietveld refinement indicate that the CFMO materials crystallize in the inverse cubic spinel phase. Temperature $(\mathrm{T}=300 \mathrm{~K}$ and $10 \mathrm{~K})$ dependent magnetization $(M(H))$ measurements indicate the long range ferromagnetic ordering in $\mathrm{CoFe}_{2-\mathrm{x}} \mathrm{Mn}_{\mathrm{x}} \mathrm{O}_{4}(x=0.00-0.15)$ ferrites. The cubic anisotropy constant $\left(K_{1}(T)\right)$ and saturation magnetization $\left(M_{s}(T)\right)$ were derived by using the "law of approach" to saturation that describes the field dependence of $M(H)$ for magnetic fields much higher than the coercive field $\left(H_{c}\right)$. Saturation magnetization $\left(M_{s}\right)$, obtained from the model, decreases with increasing temperature. For $\mathrm{CoFe}_{2} \mathrm{O}_{4}, M_{s}$ decreases from $3.63 \mu_{\mathrm{B}}$ per formula unit (f.u.) to $3.47 \mu_{\mathrm{B}} /$ f.u. with increasing temperature from 10 to $300 \mathrm{~K}$. CFMO $(0.00-0.15)$ exhibit the similar trend while the magnitude of $M_{s}$ is dependent on Mn-concentration. $M_{s}$-T functional relationship obeys the Bloch's law. The lattice parameter and magnetic moment calculated for CFMO reveals that Mn ions occupying the Fe and Co position at the octahedral site in the inverse cubic spinel phase. The structure and magnetism in CFMO are further corroborated by bond length and bond angle calculations. The dielectric constant dispersion of CFMO in the frequency range of $20 \mathrm{~Hz}-1 \mathrm{MHz}$ fits to the modified Debye's function with more than one ion contributing to the relaxation. The relaxation time and spread factor derived from modeling the experimental data are $\sim 10^{-4} \mathrm{~s}$ and $\sim 0.35( \pm 0.05)$, respectively. (C) 2013 AIP Publishing LLC. [http://dx.doi.org/10.1063/1.4827416]
\end{abstract}

\section{INTRODUCTION}

Ferrites and their composites have been extensively studied in recent years due to their remarkable electrical, magnetic, and magneto-electric properties, which can be utilized in a wide range of technological applications. ${ }^{1-30}$ Ferrites find application in microwave devices, high density magnetic information storage, and biomedical devices. ${ }^{1-9}$ Among the spinel ferrites, cobalt ferrite $\mathrm{CoFe}_{2} \mathrm{O}_{4}(\mathrm{CFO})$ is a most versatile and hard ferrimagnetic material, which exhibits unique properties such as high Curie temperature, high coercivity (5400 Oe), high magnetocrystalline anisotropy, and moderate saturation magnetization $(80 \mathrm{emu} / \mathrm{g})$ at room temperature, large Kerr effect, and Faraday rotation. ${ }^{1,5-7}$ The presence of large magnetocrystalline anisotropy with a reasonable magnetization value makes $\mathrm{CFO}$ as a promising hard magnetic and recording material. The first order magnetocrystalline anisotropy $\left(\mathrm{K}_{1}\right)$ energy constant for $\mathrm{CFO}$ is $\sim 10^{6} \mathrm{erg} / \mathrm{cm}^{3}$, which is the highest for ferrites. ${ }^{8}$ Magnetostrictive CFO composites hold promise for utilization in advanced magnetomechanical stress and torque sensors because of their large

\footnotetext{
a) Author to whom correspondence should be addressed. Electronic mail: rvchintalapalle@utep.edu
}

strain derivative $(\mathrm{d} \lambda / \mathrm{dH})_{\sigma}$ and their high sensitivity of magnetization to applied stress $(\mathrm{dB} / \mathrm{d} \sigma)_{\mathrm{H}} \cdot{ }^{9}$

In Co ferrite, relatively larger oxygen ions form FCC lattice. The interstitial sites namely tetrahedral (A) and octahedral (B) sites formed by oxygen ions are occupied by metal ions. ${ }^{10-12} \mathrm{~A}$ site is occupied by the $\mathrm{Fe}^{3+}$ ions, and the $\mathrm{B}$ site is occupied by $\mathrm{Fe}^{3+}$ and $\mathrm{Co}^{2+}$ in equal proportions. In general, inverse spinel structure allows the substitution of different transition metal ions and rare earth ions as dopants, which can affect the structural, magnetic, and dielectric properties considerably. ${ }^{13-21}$

Currently, there has been an increasing interest in CFO doped with other metal ions. Efforts were directed for doping and/substituting metal ions at Co or Fe sites. Such materials offer the ability to tailor the structural, electrical, magnetic, and dielectric properties in controlled manner. For instance, partial replacement of $\mathrm{Fe}^{3+}$ by rare earth or large ionic radius ion in the spinel structure has been reported to lead to structural distortion which induces strain and significantly modifies the electrical and dielectric properties. ${ }^{12-14}$ Substitution of rare earth ions for Fe ion in Co ferrite is reported to enhance the coercivity and dielectric constant. ${ }^{22-24}$ At the same time, a decrease in saturation magnetization $\left(M_{s}\right)$ and Curie temperature $\left(T_{c}\right)$ values were reported due to the effect of foreign atoms in $\mathrm{CFO} .^{25-29}$ 
Several reports are also available on the effect of manganese (Mn) ions incorporated into CFO. Melikhov et al., ${ }^{30}$ Paulsen et al., ${ }^{31}$ and Caltun et al. ${ }^{32}$ have reported that the substitution of $\mathrm{Mn}$ for $\mathrm{Fe}$ ions in $\mathrm{Co}$ ferrite results in decreasing the $M_{s}$ and $T_{c}$ values while the magnetostriction values are retained unaffected. Decreasing anisotropy constant $\left(K_{1}\right)$ with increasing $\mathrm{Mn}$ concentration was also reported. ${ }^{30-32}$ However, most of the existing reports focus on the high end of $\mathrm{Mn}$ concentration, i.e., $x=0.1-0.5$ in $\mathrm{CoFe}_{2-\mathrm{x}} \mathrm{Mn}_{\mathrm{x}} \mathrm{O}_{4}$ (CFMO). In addition, in most of the cases, $x$ values were increased in steps of 0.1 , where the information on the structural distortion and property changes due to fractional amount of dopant are often missing. However, on the other hand, doping or substituting metal ions at lower concentration level $(x \leq 0.1)$ and elucidating the underlying physics are important since the effect of foreign atoms is most remarkable at the very first, lower-concentration incorporation into the bulk of CFO. Recently, we demonstrated that such remarkable effects will occur at very low concentration of the metal ions substituted for $\mathrm{Fe}$ in $\mathrm{Co}$ - and/or Ni-based ferrites. Furthermore, in the case of $\mathrm{Mn}$ ions into CFO, either structure-magnetic property and/or structure-electric property alone are considered for investigation. As such, a comprehensive understanding of the effect of Mn-ions on the crystal structure, composition, magnetic, and dielectric properties is missing, especially for doping ions with very low concentration. In this work, we have performed a systematic investigation of CFMO compounds with variable Mn composition ( $x$ ) in the range of $0.00-0.15$. Attention has been paid to evaluate the effect of $\mathrm{Mn}$ ions on the structural, magnetic and dielectric properties. The results obtained are presented and discussed in this paper to establish a correlation between structure, magnetic, and dielectric properties in $\mathrm{CoFe}_{2-\mathrm{x}} \mathrm{Mn}_{\mathrm{x}} \mathrm{O}_{4}$ materials.

\section{EXPERIMENTAL DETAILS}

The CFMO polycrystalline compounds were prepared from $99.99 \%$ pure $\mathrm{CoO}, \mathrm{Fe}_{2} \mathrm{O}_{3}$, and $\mathrm{Mn}_{2} \mathrm{O}_{3}$ by the solid state reaction method. Powders of the starting materials were ground in a mortar and pestle for $1 \mathrm{~h}$ and the mixtures were heat treated in air at $1200^{\circ} \mathrm{C}$ for $12 \mathrm{~h}$ employing controllable furnace with the heating rate of $200^{\circ} \mathrm{C}$ per hour. Phase identification and crystal structure of the materials synthesized were investigated using X-ray diffraction (XRD) measurements employing a Bruker D8 Discover X-ray diffractometer. Measurements were made at room temperature using $\mathrm{Cu}$ $\mathrm{K} \alpha$ radiation $(\lambda=1.5406 \AA)$. Structural analysis was carried out employing Rietveld refinement technique using the General Structure Analysis System (GSAS) program. ${ }^{15}$ The procedure employed to calculate the bond angles and bond lengths is outlined elsewhere. ${ }^{16}$ Temperature and field dependent magnetization had been measured using a Quantum Design superconducting quantum interference device (SQUID) magnetometer in magnetic fields from $-6 \mathrm{kOe}$ to $+6 \mathrm{kOe}$ and temperatures from $10 \mathrm{~K}$ to $300 \mathrm{~K}$. Dielectric measurements were carried out employing a LCR meter (HP 4284 A). For dielectric measurements, silver was used as an electrode. Highly conducting silver paste was applied on both sides of the pellet and dried before the measurements were carried out. Pellets ( $1.5 \mathrm{~mm}$ thick) of CFMO were used for the impedance and dielectric measurements. AC signal of $1.2 \mathrm{~V}$ (rms) with the frequency range from $20 \mathrm{~Hz}$ to $1 \mathrm{MHz}$ was employed for all the measurements.

\section{RESULTS AND DISCUSSION}

\section{A. Structural properties}

The XRD patterns of Mn substituted and pure Co ferrites are shown in Figure 1. The calculated patterns after the Rietveld refinement carried out using the GSAS program are also shown along with the experimental XRD patterns (Fig. 1). XRD data indicate that the compounds were crystallized in inverse spinel phase corresponding to the space group $\mathrm{Fd}-3 \mathrm{~m}$ (227). $\mathrm{CoFe}_{2} \mathrm{O}_{4}$ is found to form in the inverse spinel phase without any impurity phase, and the calculated value of the lattice constant is $8.398 \AA$, which agrees with the reported standard value. ${ }^{33}$ XRD data reveal that $\mathrm{CoFe}_{2-\mathrm{x}} \mathrm{Mn}_{\mathrm{x}} \mathrm{O}_{4}(x=0.05,0.075,0.10$, and 0.15) materials also crystallize in the inverse spinel phase without any impurity or secondary phase. The calculated lattice constant value for $\mathrm{CoFe}_{2-\mathrm{x}} \mathrm{Mn}_{\mathrm{x}} \mathrm{O}_{4}(\mathrm{x}=0,0.05,0.075,0.10$, and 0.15 ) compounds were found to decrease from $8.398 \AA$ to $8.378 \AA$. The variation of lattice constant with Mn concentration $(x)$ is shown in Figure 2. It can be noted that the lattice constant values of Mn-substituted cobalt ferrite $(x=0.05-0.15)$ are not varying significantly, while there is a considerable difference in the lattice parameters of pure and $\mathrm{Mn}$-substituted $\mathrm{CoFe}_{2} \mathrm{O}_{4}$ in general. The observed lattice constant which decreases at the initial Mn substitution (5\%) into CFO can be explained based on the competing tendency of $\mathrm{Mn}^{3+}$ ions for the site occupation. Specifically,

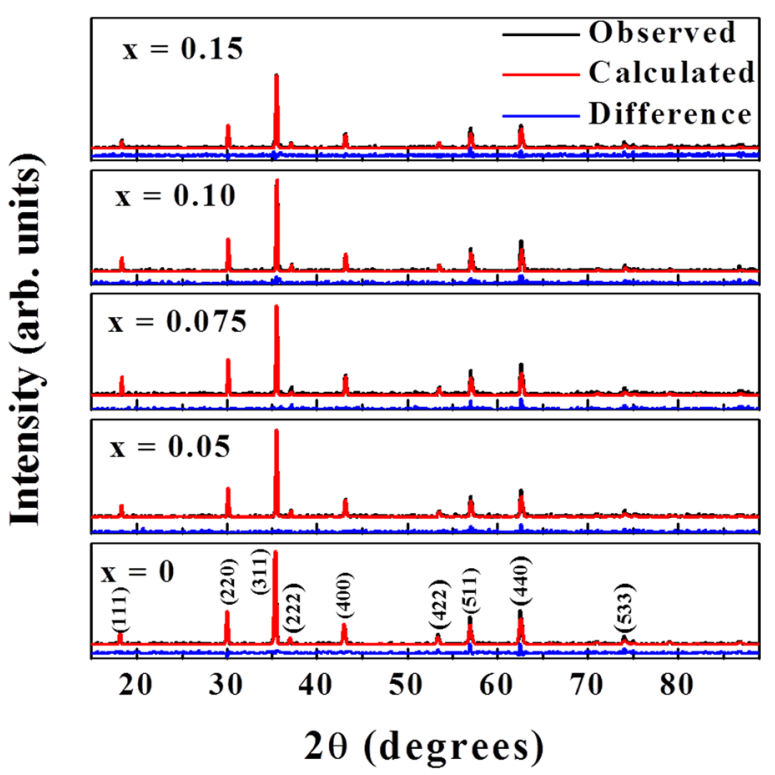

FIG. 1. XRD patterns of the CFMO ferrites as a function of $x$. Experimental XRD patterns along with those calculated from refinement procedure are shown. The difference between the experimental and calculated patterns was also shown. The data indicate that the CFMO crystallize in inverse spinel structure. 


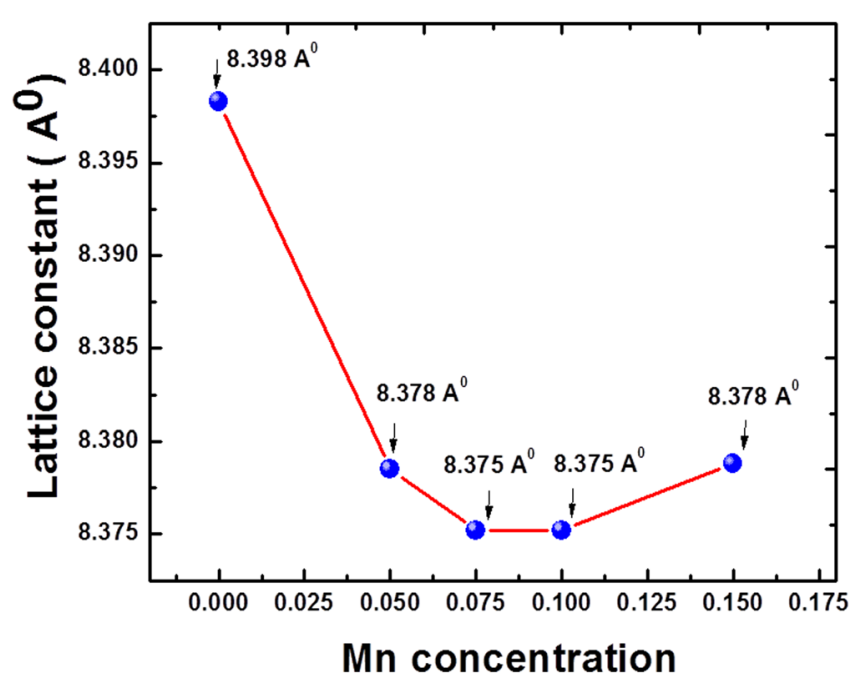

FIG. 2. Variation of lattice parameter with Mn concentration (x). Manganese substitution induced lattice contraction compared to that of pure CFO $(x=0)$ is evident.

at the initial Mn-substitution, part of $\mathrm{Mn}^{3+}(0.645 \AA)$ ions may prefer to occupy the $\mathrm{Co}^{2+}(0.745 \AA)$ at octahedral site in addition to the maximum preference of $\mathrm{Mn}^{3+}$ substituting for $\mathrm{Fe}^{3+}(0.645 \AA)$ at octahedral site. While the XRD refinement data clearly indicate the best fitting for $\mathrm{Mn}^{3+}$ ions to occupy $\mathrm{Fe}^{3+}$ at $\mathrm{B}$ site, the lattice constant decrease was also supported by bond length and bond angle calculations (Table I) derived from refinement procedure. Most importantly, evidence for $\mathrm{Mn}$ ions initially competing for $\mathrm{Co}^{2+}$ and $\mathrm{Fe}^{3+}$ ions was also supported by the observed behavior of saturation magnetization with Mn concentration as discussed below.

\section{B. Magnetic properties}

Figure 3 displays a representative field dependent magnetization $(M(H))$ data measured at $10 \mathrm{~K}, M(H)$ data also measured at $300 \mathrm{~K}$ (not shown), for pure and $\mathrm{Mn}$ substituted CFO samples. At room temperature $(300 \mathrm{~K})$ as well as at low temperature $(10 \mathrm{~K})$, all samples exhibit hysteresis loops in $M-H$ data indicating the existence of a long range ferromagnetic ordering in all pure and $\mathrm{Mn}$ substituted $\mathrm{CoFe}_{2-\mathrm{x}} \mathrm{Mn}_{\mathrm{x}} \mathrm{O}_{4}$ ferrites. The hysteresis loops open at low fields and magnetization seems to approach to saturation above $10 \mathrm{kOe}$, especially for Mn substituted samples. The coercive field $\left(H_{c}\right)$ as well as remnant magnetization $\left(M_{r}\right)$ is

TABLE I. Bond angle and bond length determined for $\mathrm{CoFe}_{2-\mathrm{x}} \mathrm{Mn}_{\mathrm{x}} \mathrm{O}_{4}$ ferrites using the refinement of XRD data.

\begin{tabular}{|c|c|c|c|}
\hline Composition & $\begin{array}{c}\mathrm{Fe}^{3+}-\mathrm{O}^{2-} \mathrm{Fe}^{3+} \\
\left(\mathrm{Mn}^{3+}\right) \\
\text { Bond angle (deg) }\end{array}$ & $\begin{array}{c}\mathrm{Co}^{2+}-\mathrm{O}^{2-} \mathrm{Co}^{2+} \\
\text { Bond angle } \\
(\mathrm{deg})\end{array}$ & $\begin{array}{c}\mathrm{O}^{2-} \mathrm{Fe}^{3+} \\
\left(\mathrm{Mn}^{3+}\right) \\
\text { Bond length }(\AA)\end{array}$ \\
\hline $\mathrm{CoFe}_{2} \mathrm{O}_{4}$ & 159.2 & 90.6 & 2.05 \\
\hline $\mathrm{CoFe}_{1.95} \mathrm{Mn}_{0.05} \mathrm{O}_{4}$ & 157.1 & 90 & 2.045 \\
\hline $\mathrm{CoFe}_{1.925} \mathrm{Mn}_{0.075} \mathrm{O}_{4}$ & 157.3 & 89.9 & 2.043 \\
\hline $\mathrm{CoFe}_{1.90} \mathrm{Mn}_{0.10} \mathrm{O}_{4}$ & 157.4 & 89.8 & 2.041 \\
\hline $\mathrm{CoFe}_{1.85} \mathrm{Mn}_{0.15} \mathrm{O}_{4}$ & 157.4 & 89.8 & 2.042 \\
\hline
\end{tabular}

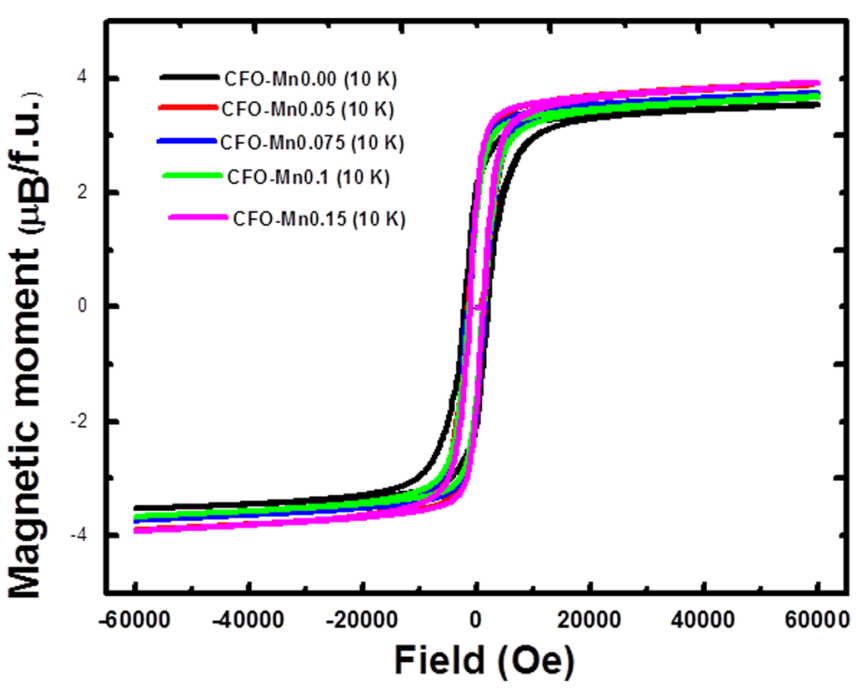

FIG. 3. Field dependent magnetization $M(H)$ curves obtained at $10 \mathrm{~K}$ for $\mathrm{CoFe}_{2-x} \mathrm{Mn}_{x} \mathrm{O}_{4}$ ferrites.

strongly dependent on Mn concentration as well as temperature. The $H_{c}$ of all samples increases with decreasing temperature. For instance, the $H_{c}$ of pure cobalt ferrite sample increases from 200 to 2165 Oe, going from 300 to $10 \mathrm{~K}$. With Mn substitution, the $H_{c}$ initially decreases sharply with small amount of $\mathrm{Mn}$ substitution and then remains more or less same with further doping. For example, $H_{c}$ from $2160 \mathrm{Oe}$ (at $10 \mathrm{~K})$ decreases to $1300 \mathrm{Oe}(300 \mathrm{~K})$ with $5 \% \mathrm{Mn}$ doping and it remains same with further doping. This is an indication that $\mathrm{Fe}$ is being replaced by $\mathrm{Mn}$ ions in the $\mathrm{CoFe}_{2-\mathrm{x}} \mathrm{Mn}_{\mathrm{x}} \mathrm{O}_{4}$ mixed ferrite.

The temperature dependence of magnetization from $10 \mathrm{~K}$ to $300 \mathrm{~K}$ is obtained under two magnetic fields $5 \mathrm{kOe}$ and $50 \mathrm{kOe}$. Figure 4 shows the temperature dependent magnetization $M(T)$ data taken under $50 \mathrm{kOe}$ for all pure and Mn doped CFO samples. The field cooled (FC) magnetization strongly depends on the applied magnetic field, especially at lower temperatures. For instance, under $5 \mathrm{kOe}$ (not shown), the magnetization of all samples increases with increasing temperature from $10 \mathrm{~K}$ to $300 \mathrm{~K}$. At higher magnetic field of $50 \mathrm{kOe}$, the magnetization increases initially with increasing temperature showing maximum and then decreases with further increase of temperature. Similar behavior has already been observed in CFO and other ferrimagnetic materials. ${ }^{34}$ It is well known that in a ferrimagnetic material each sub lattice is spontaneously magnetized and the two sub lattice magnetizations are opposed to each other. The net magnetization in a ferrimagnetic material is given by $M_{A}-M_{B}$, where $M_{A}$ and $M_{B}$ are the magnetization of sub lattices $\mathrm{A}$ and $\mathrm{B}$, respectively. If the magnetization of one sub lattice decreases less rapidly with increasing temperature than the other one, the resultant magnetization increases with temperature and goes through a maximum before finally dropping to zero. The magneto-crystalline anisotropy of cobalt ferrite is so high that it prevents complete approach to saturation at the magnetic field available in our SQUID magnetometer. The use of the "Law of Approach (LA)" is more useful to determine the temperature dependent 
magneto-crystalline anisotropy constant $K_{1}(T)$ and temperature dependent saturation magnetization $M_{s}(T)$, which is discussed later in this section.

The temperature dependence of the cubic anisotropy constant $K_{1}(T)$ and saturation magnetization $M_{s}(T)$ for all samples were estimated by a "LA" to saturation that describes the field dependent of the magnetization for magnetic field much higher than the coercive field $\left(H_{c}\right)$. The magnetization near $M_{S}$ for randomly oriented polycrystalline samples with a cubic anisotropy can be written by the following equation: ${ }^{10}$

$$
M=M_{s}\left(1-\frac{8 K_{1}^{2}}{105 \mu_{0}^{2} M_{s}^{2} H^{2}}\right)+c H,
$$

where $M$ is the magnetization, $H$ is the applied magnetic field, $M_{s}$ is the saturation magnetization, $\mu_{0}$ is the magnetic permeability in free space, and $c$ is linear constant term. The phenomenological linear term $c H$ is very small at high magnetic field. To use the minimum number of parameters, this linear term is neglected in finding $M_{s}(T)$ and $K_{1}(T)$. The temperature dependence of $K_{1}(T)$ and $M_{s}(T)$ is obtained by fitting Eq. (1) to the values of magnetization corresponding to the high magnetic field part of the curve, e.g., using values of $H$ above which the hysteresis loop is completely closed. For the fitting process, only magnetization data above 50 kOe are used. Representative fitted curves of $M_{s}$ to LA at room temperature and $10 \mathrm{~K}$ for pure $\mathrm{CFO}$ sample are shown in Figure 5.

The temperature and substitution dependence of $K_{1}$ obtained by fitting Eq. (1) are shown in Fig. 6(a). We found that $K_{1}$ is strongly dependent on temperature. For instance, at lower temperatures $(10 \mathrm{~K})$, it is much higher than at higher temperature $(300 \mathrm{~K})$. For example, for pure CFO sample at room temperature, the value of $K_{1}$ is $8.2 \times 10^{6} \mathrm{erg} / \mathrm{cm}^{3}$ and it increases to $\left(1.6 \times 10^{7}\right) \mathrm{erg} / \mathrm{cm}^{3}$ with decreasing temperature at $10 \mathrm{~K}$. This is an indication that the magnetic field is no longer enough to align all spins at low temperature. These

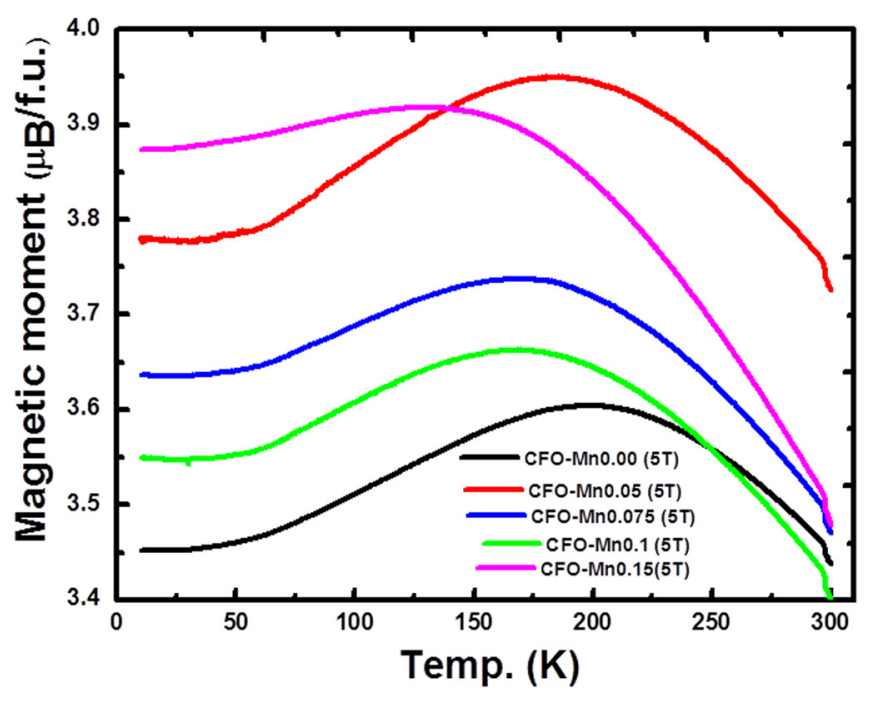

FIG. 4. Temperature dependent magnetization $M(T)$ for $\mathrm{CoFe}_{2-x} \mathrm{Mn}_{x} \mathrm{O}_{4}$. The data are obtained at temperatures from 10 to $300 \mathrm{~K}$ at a field of $50 \mathrm{kOe}$.

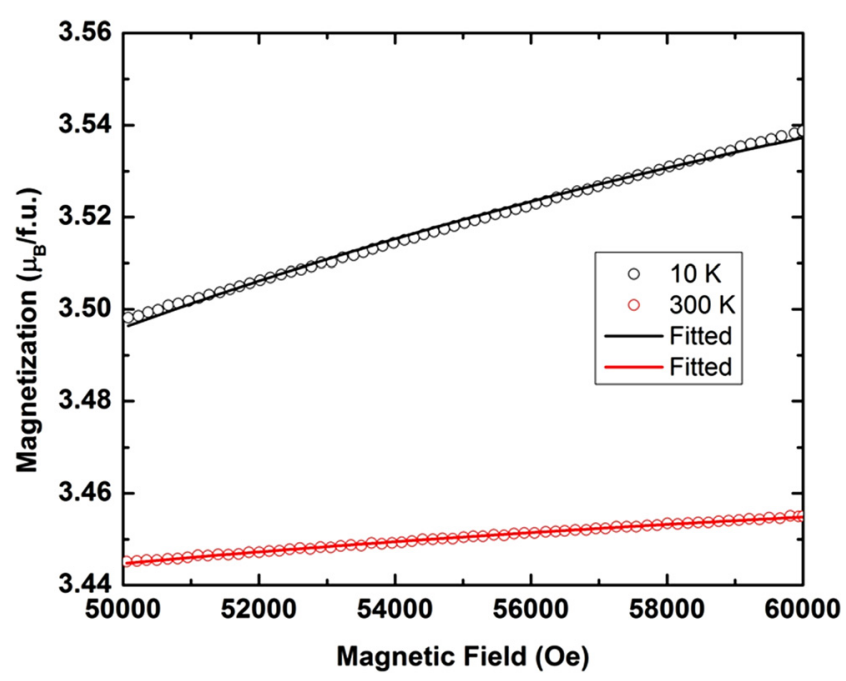

FIG. 5. Fitted data of $M_{s}$ to Law of Approach to saturation for pure CFO at $10 \mathrm{~K}$ and $300 \mathrm{~K}$.

results are similar to those reported by Melikhov et al. ${ }^{30}$ The high $K_{l}$ value was expected because the strong anisotropy of cobalt ferrite is primarily due to the presence of $\mathrm{Co}^{2+}$ ions on the octahedral sites of the spinel structure. At lower temperature, $K_{1}$ slightly increases with Mn substitution and it is less sensitive with $\mathrm{Mn}$ substitution at room temperature. Atif et $a l .{ }^{35}$ reported that the value of $K_{1}$ for the samples decreases with $\mathrm{Mn}$ substitution in $\mathrm{Co}_{1-\mathrm{x}} \mathrm{Mn}_{\mathrm{x}} \mathrm{Fe}_{2} \mathrm{O}_{4}$ where Mn mainly replaces Co ions on the octahedral-sites, which is responsible for a high value of the anisotropy in cobalt ferrite. In our case, most of the $\mathrm{Mn}$ ions replaces $\mathrm{Fe}$ ions on the octahedral-sites and some $\mathrm{Mn}^{3+} / \mathrm{Mn}^{2+}$ ions go into the $\mathrm{Co}^{2+}$ ions on the octahedral-sites.

To better understand the magnetic behavior of $\mathrm{Mn}$ doped CFO, next we discuss the variation of coercivity $H_{c}$ as a function of Mn concentration. Fig. 6(b) shows the variation of $H_{c}$ with Mn substitution. With Mn substitution, the $H_{c}$ initially decreases sharply with small amount of Mn substitution and then remains more or less same with further doping. The $H_{c}$ of a hard ferromagnetic/ferrimagnetic materials is given by a simple model as suggested by Kronmüller, ${ }^{36}$

$$
H_{c}=\frac{\alpha\left(2 K_{1}+l_{s} \sigma\right)}{\mu_{0} M_{s}}-D \cdot M_{s},
$$

where the parameters $K_{1}, l_{\mathrm{S}}, \mu_{0}$, and $M_{S}$ are the same parameters with Eq. (1), whereas the parameters $\alpha, D$, and $\sigma$ are the microstructural parameter, effective demagnetization factor, and the internal stress, respectively. The internal stress is close to zero for bulk ferrite ceramic materials. Here, the first term gives a rough/approximate proportionality to an effective anisotropy field, while the second term $\left(D \cdot M_{S}\right)$ describes an average stray field due to the neighboring grains. For estimating the microstructural parameter $\alpha$ which describes the percentage of the anisotropy field that contributes to the coercivity, the second term is neglected and $\alpha$ can be calculated from the relation $\alpha=\mu_{0} M_{s} H_{c} / 2 K_{1}$. Figure $6(c)$ shows the variation of microstructural parameter $(\alpha)$ 

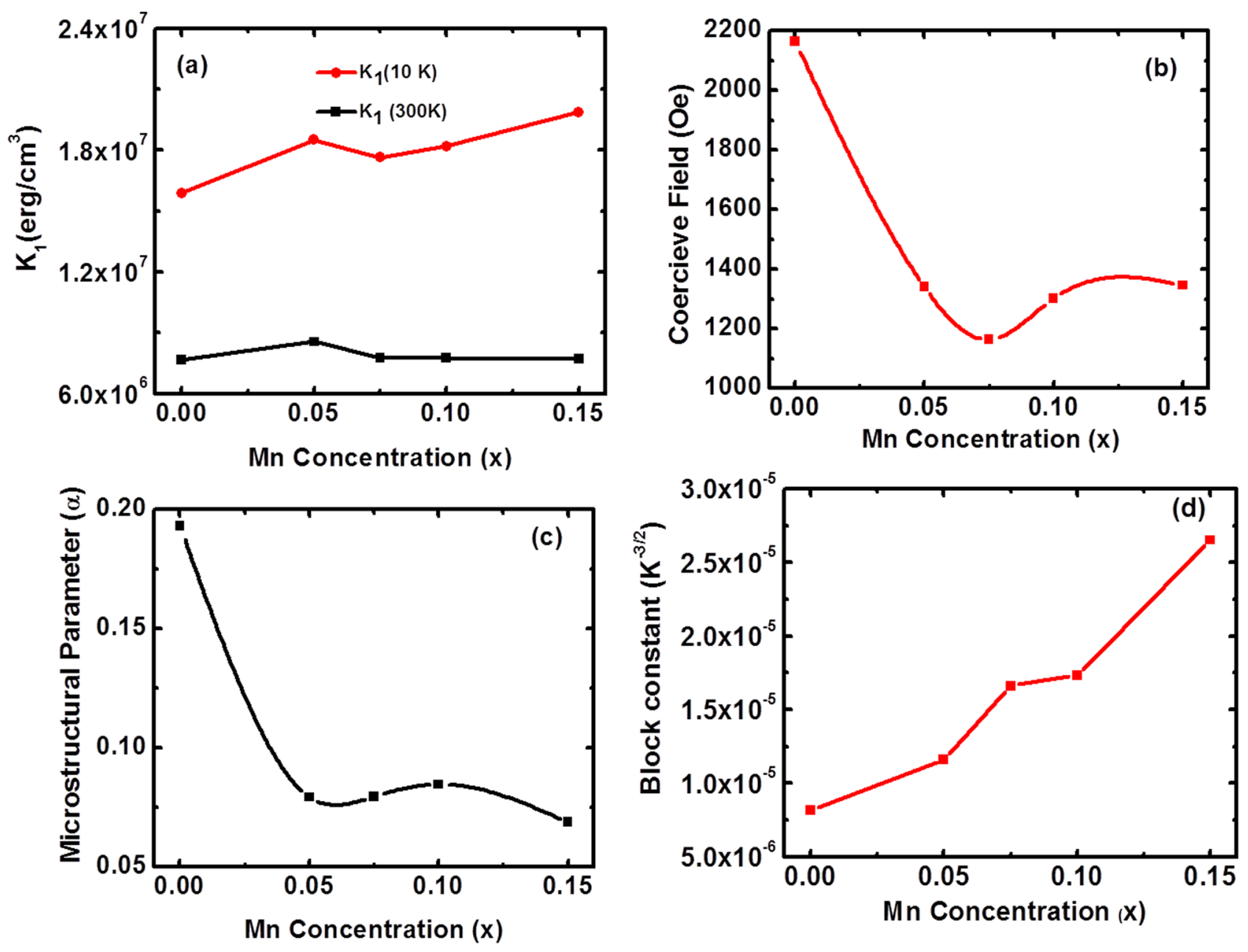

FIG. 6. (a) Variation of anisotropy constant $\left(\mathrm{K}_{1}\right)$ with Mn concentration at $10 \mathrm{~K}$ and $300 \mathrm{~K}$; (b) variation of coercive field (Hc) with Mn concentration; (c) variation of the microstructural parameter $(\alpha)$ with Mn concentration; (d) variation of Bloch constant with Mn concentration.

with Mn substitution. It is interesting to see that $H_{C}$ and $\alpha$ follow the same trend where with Mn substitution both the parameters decrease sharply with small Mn substitution and then it remains more or less constant. This means that the substitution dependence of the coercivity can be better understood regarding the strongly changing microstructure with $x$. Hence, intrinsic parameters such as $\mathrm{M}_{\mathrm{S}}$ and/ $\mathrm{K}_{1}$ play only a minor role in the present case.

The saturation magnetization $\left(M_{S}\right)$ obtained by fitting Eq. (1) decreases with increasing temperature. For instance, for pure CFO sample, $M_{s}$ is $3.63 \mu_{\mathrm{B}} /$ f.u. and $3.47 \mu_{\mathrm{B}} /$ f.u. at $10 \mathrm{~K}$ and $300 \mathrm{~K}$, respectively. The temperature dependence of the saturation magnetization, $M_{s}$, can be well fitted to Bloch's law. Bloch's law describes the temperature-dependence of the saturation magnetization $M_{s}$ of ferromagnetic materials where the temperature-dependent decrease in $M_{s}$ is dominated by the excitation of long wavelength spin-wave fluctuations, called magnons. At low temperatures, the first-order term of Bloch's law can be written as

$$
M_{s}(T)=M_{s}(0)\left[1-b T^{c}\right]
$$

where $M_{s}(T)$ is the temperature-dependent saturation magnetization, $M_{s}(0)$ is the magnetization of the ground state at
$\mathrm{T}=0 \mathrm{~K}, \mathrm{~b}$ is Bloch's constant, and $\mathrm{c}$ is the Bloch exponent. Bloch's law indicates that the decrease in the saturation magnetization with increasing temperature due to spin-wave excitations is described by a power law in T. Assuming the exponent $\mathrm{c}=1.5$, the Bloch constant " $\mathrm{b}$ " for all samples determined using Bloch's law is shown in Figure 6(d). It is clear from the plot that $\mathrm{b}$ increases continuously with increasing Mn concentration. The increase of Bloch's constant b with $\mathrm{Mn}$ substitution indicates that the spontaneous magnetization decreases faster with the temperature in doped samples than pure CFO.

The observed saturation magnetization $M s(0)$ at $0 \mathrm{~K}$ for pure $\mathrm{CFO}$ is $3.63 \mu_{\mathrm{B}} /$ f.u. which is very close to theoretical value of $3.87 \mu_{\mathrm{B}}$ for an ideal cubic spinel structure. With Mn substitution in $\mathrm{CoFe}_{2-\mathrm{x}} \mathrm{Mn}_{\mathrm{x}} \mathrm{O}_{4}$, the $M s(0)$ increases sharply with low Mn substitution of 5\%, then decreases slowly till Mn substitution of $10 \%$ and again it increases with further increase of Mn concentration. The substitution dependence of saturation magnetization $M_{S}(0)$ can be better understood on the basis of crystal structure of CFO. CFO is a ferrimagnetic material having cubic inverse spinel structure with oxygen anions forming a fcc closed packing and $\mathrm{Fe}^{3+}$ cations located at the interstitial tetrahedral sites and octahedral sites and $\mathrm{Co}^{2+}$ cations located at the octahedral sites. The net 


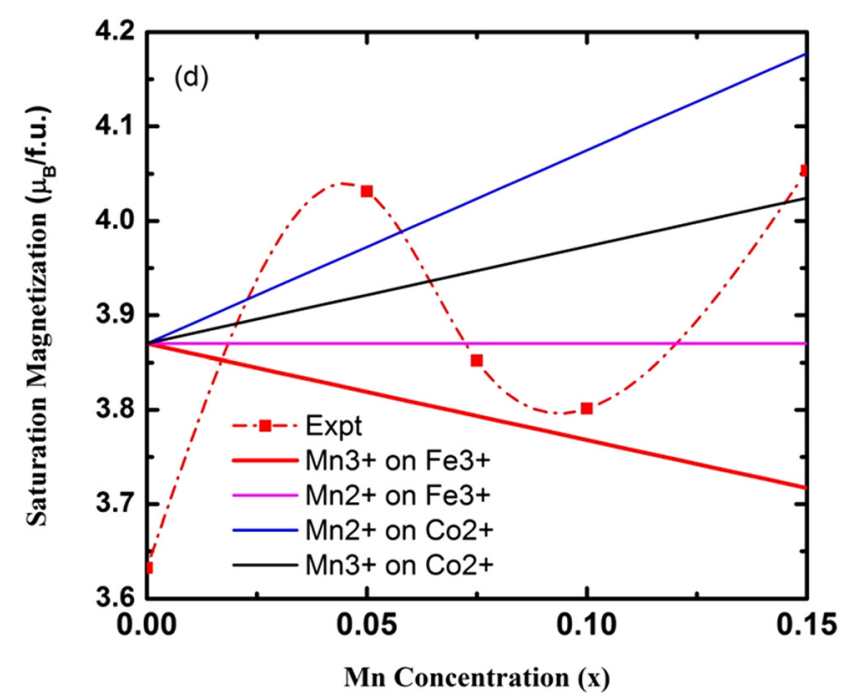

FIG. 7. Variation of saturation magnetization $\left(M_{s}\right)$ with Mn concentration $(x)$ in $\mathrm{CoFe}_{2-x} \mathrm{Mn}_{x} \mathrm{O}_{4}$.

magnetic moment of the unit cell of pure CFO comes only from $\mathrm{Co}^{2+}$ ions with a magnetic moment of $3.87 \mu_{\mathrm{B}} /$ f.u. $\mathrm{Mn}^{2+}$ and/or $\mathrm{Mn}^{3+}$ ions can replace $\mathrm{Co}^{2+}$ ions and $\mathrm{Fe}^{3+}$ ions

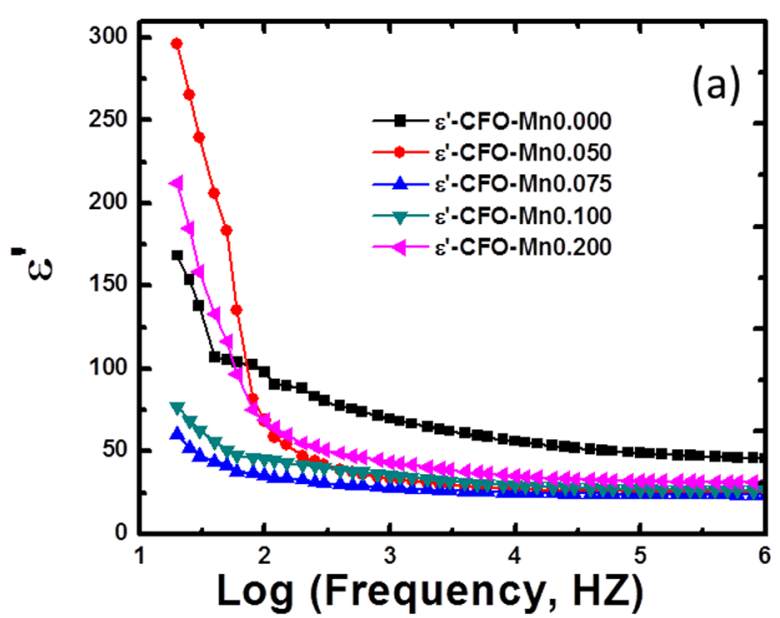

located at the octahedral sites in four possible ways: (1) $\mathrm{Mn}^{2+}$ on $\mathrm{Co}^{+2}$, (2) $\mathrm{Mn}^{3+}$ on $\mathrm{Co}^{+2}$, (3) $\mathrm{Mn}^{3+}$ on $\mathrm{Fe}^{3+}$, and (4) $\mathrm{Mn}^{2+}$ on $\mathrm{Fe}^{3+}$ ions. The variation of calculated $M_{s}(0)$ with Mn for these four configurations is shown in Fig. 7. The calculation shows that the $M_{s}(0)$ does not change for case (4), decreases for case (3), and increases for both cases (1) and (2) with Mn substitution. Experimental data indicates that initially $\mathrm{Mn}^{3+} / \mathrm{Mn}^{2+}$ goes to $\mathrm{Co}^{2+}$ site and then it goes to $\mathrm{Fe}^{3+}$ sites. This is also in agreement with XRD data, particularly the variation of lattice parameter with Mn substitution in CFO.

\section{Dielectric properties}

The frequency dependence of the $\varepsilon^{\prime}$ (real part of dielectric constant), $\varepsilon^{\prime \prime}$ (imaginary part of dielectric constant), and $\tan \delta$ (loss tangent) of pure and Mn doped CFO is measured at $300 \mathrm{~K}$ in the frequency range $20 \mathrm{~Hz}-1 \mathrm{MHz}$ (Figs. 8(a)-8(c)). It is clear from Fig. 8 that the $\varepsilon^{\prime}, \varepsilon^{\prime \prime}$, and $\tan \delta$ are strongly dependent on the frequency. The $\varepsilon^{\prime}, \varepsilon^{\prime \prime}$, and $\tan \delta$ initially showed a sharp decrease with increase in frequency up to $1 \mathrm{kHz}$ and then decreased slowly and become almost constant up to $1 \mathrm{MHz}$, indicating the usual dielectric dispersion.
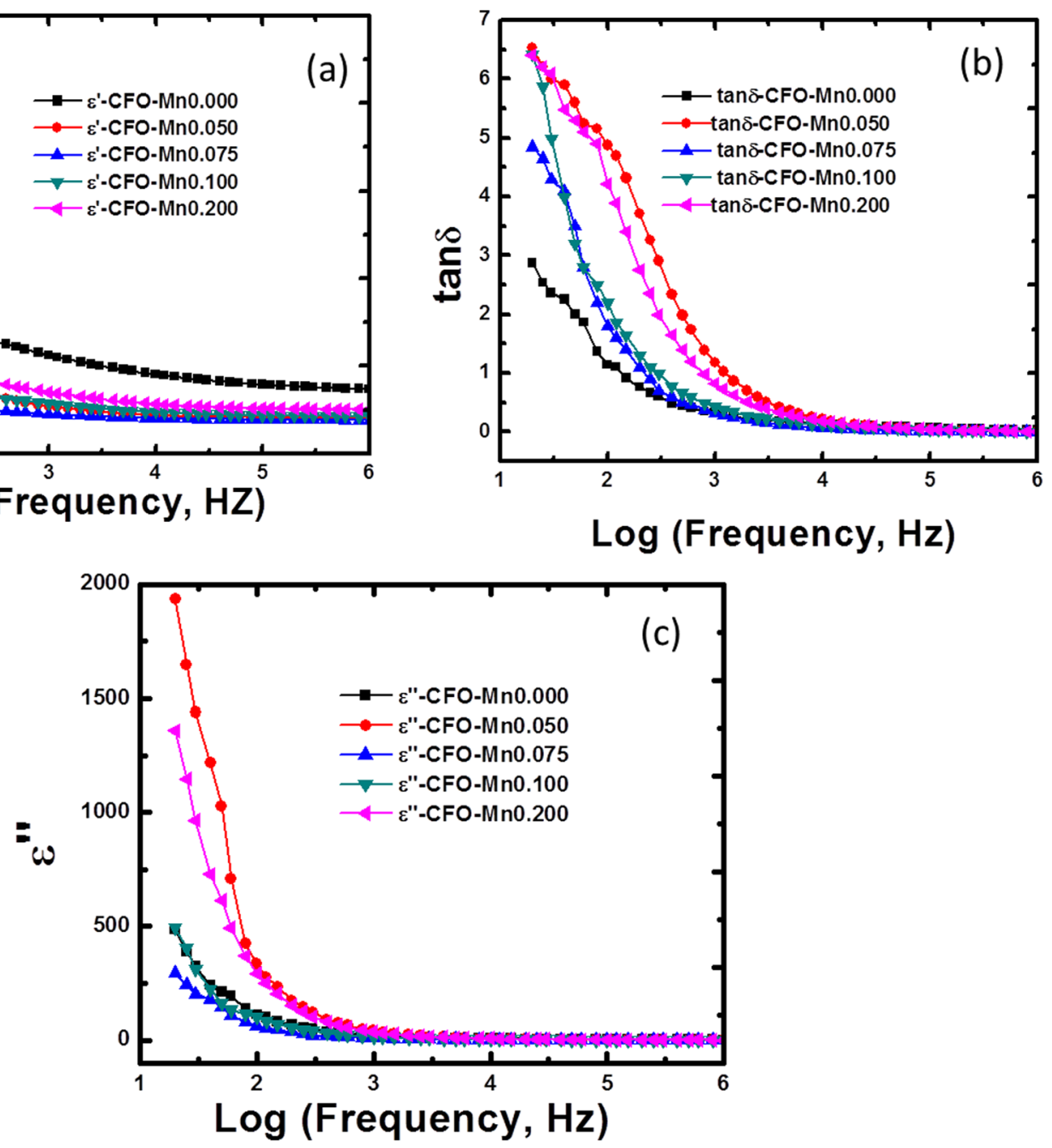

FIG. 8. Room temperature frequency dependent: (a) real part of dielectric constant $\left(\varepsilon^{\prime}\right)$, (b) loss tangent ( $\tan \delta$ ), and (c) the imaginary part of dielectric constant $\left(\varepsilon^{\prime \prime}\right)$. 


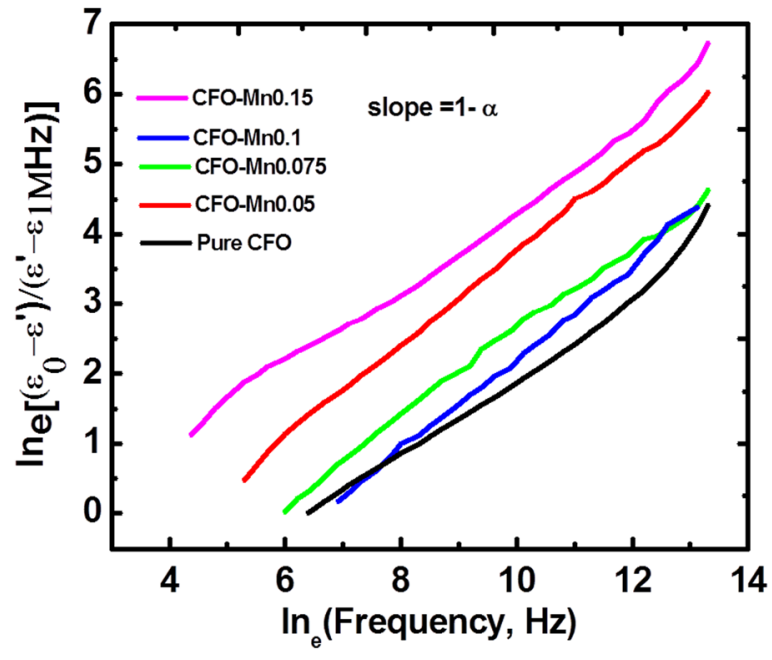

FIG. 9. Plots of $\ln _{\mathrm{e}}\left[\left(\varepsilon_{0}^{\prime}-\varepsilon^{\prime}\right) /\left(\varepsilon^{\prime}-\varepsilon^{\prime}{ }_{1 \mathrm{MHz}}\right)\right]$ with $\ln _{\mathrm{e}}(f)$. The spread factor is calculated from the slope of the linear plots.

The dispersion of dielectric constant with frequency is due to Maxwell-Wagner type interfacial polarization in agreement with Koop's phenomenological theory. ${ }^{37-39}$ It is also well known that the polarization in ferrite is through a mechanism similar to the conduction process. ${ }^{40}$ The presences of $\mathrm{Fe}^{3+}$ and $\mathrm{Fe}^{2+}$ ions have rendered ferrite materials dipolar. Rotational displacement of dipoles results in orientational polarization. In ferrites, rotation of $\mathrm{Fe}^{2+} \leftrightarrow \mathrm{Fe}^{3+}$ and $\mathrm{Mn}^{2+} \leftrightarrow \mathrm{Mn}^{3+}$ dipoles may be visualized as the exchange of electrons between the ions so that the dipoles align themselves in response to the alternating field. The existence of inertia to the charge movement would cause relaxation of the polarization. The polarization at lower frequencies may results from electron hopping between $\mathrm{Fe}^{3+} \leftrightarrow \mathrm{Fe}^{2+}$ and $\mathrm{Mn}^{2+} \leftrightarrow \mathrm{Mn}^{3+}$ ions in the ferrite lattice. The polarization decreases with increase in frequency and reaches a constant value due to the fact that beyond a certain frequency of external field the electron exchange $\mathrm{Fe}^{3+} \leftrightarrow \mathrm{Fe}^{2+}$ and $\mathrm{Mn}^{2+} \leftrightarrow \mathrm{Mn}^{3+}$ cannot follow the alternating field. ${ }^{41}$ In the present CFMO system, the presence of $\mathrm{Co}^{3+} / \mathrm{Co}^{2+}$ ions gives rise to p-type carriers. The local displacement of p-type carriers in the direction of an external electric field also contributes to net polarization in addition to that of the n-type carriers. However, the contribution due to p-type carriers should be smaller than the electronic exchange between $\mathrm{Fe}^{3+} / \mathrm{Fe}^{2+}$ and $\mathrm{Mn}^{3+} / \mathrm{Mn}^{2+}$ and has an opposite sign. Since the mobility of p-type carriers is smaller than n-type carriers, their contribution to the polarization decreases more rapidly even at lower frequencies. As a result, initially, the net polarization decreases sharply at lower frequency and then decreases slowly at higher frequency (above $1 \mathrm{kHz}$ ), as is observed in the present system. Similar results were reported earlier by Popandian et al. . $^{41}$

In general, the dielectric loss in polycrystalline ferrites is a result of the lag in polarization with respect to the applied alternating electric field. Moreover, the loss factor is considered to be caused by domain wall resonance. At higher frequencies, losses are found to be low since domain wall
TABLE II. Spreading factor $(\alpha)$ and the relaxation time $(\tau)$ determined for $\mathrm{CoFe}_{2-\mathrm{x}} \mathrm{Mn}_{\mathrm{x}} \mathrm{O}_{4}$ ferrites from modeling the data obtained from experiment.

\begin{tabular}{lcc}
\hline \hline Composition & Spreading factor $(\alpha)$ & Relaxation time (S) \\
\hline $\mathrm{CoFe}_{2} \mathrm{O}_{4}$ & 0.42 & $1.74 \times 10^{-4}$ \\
$\mathrm{CoFe}_{1.95} \mathrm{Mn}_{0.05} \mathrm{O}_{4}$ & 0.34 & $1.14 \times 10^{-3}$ \\
$\mathrm{CoFe}_{1.925} \mathrm{Mn}_{0.075} \mathrm{O}_{4}$ & 0.40 & $2.24 \times 10^{-4}$ \\
$\mathrm{CoFe}_{1.90} \mathrm{Mn}_{0.10} \mathrm{O}_{4}$ & 0.33 & $1.52 \times 10^{-4}$ \\
$\mathrm{CoFe}_{1.85} \mathrm{Mn}_{0.15} \mathrm{O}_{4}$ & 0.43 & $2.10 \times 10^{-4}$ \\
\hline \hline
\end{tabular}

motion is inhibited and magnetization is forced to change by rotation. $^{42}$

The dispersion of $\varepsilon^{\prime}$ can also be explained based on the contributions from various sources of polarizations. ${ }^{43,44}$ The larger value of $\varepsilon^{\prime}$ at lower frequencies is mainly due to the contributions from polarizations of ionic, space charge/interface, and grain-boundaries. The decrease (and disappearance finally) in ionic and orientation polarizability with increasing frequency may be responsible for the decrease in $\varepsilon^{\prime}$ at higher frequencies. ${ }^{45}$ Since more than one ion $\left(\mathrm{O}^{2-}, \mathrm{Fe}^{3+}, \mathrm{Co}^{2+}\right.$, and $\mathrm{Mn}^{3+}$ ions) contributes to the relaxation process, the data were fitted to the modified Debye's function that considers the possibility of more than one ion contributing to the relaxation. ${ }^{43,44}$ Using this model, the observed dispersion of the dielectric constant can be modeled using the equation

$$
\varepsilon^{\prime}=\varepsilon_{\infty}^{\prime}+\frac{\left(\varepsilon_{0}^{\prime}-\varepsilon_{\infty}^{\prime}\right)}{\left[1+(\omega \tau)^{2(1-\alpha)}\right]},
$$

where $\varepsilon^{\prime}$ is the real part of the dielectric constant, $\varepsilon_{\infty}^{\prime}$ is the dielectric constant at higher frequency (at $1 \mathrm{MHz}$ ), $\varepsilon_{0}^{\prime}$ is the dielectric constant at lowest possible frequency (in our case $20 \mathrm{~Hz}), \omega=2 \pi f$ is the angular frequency with $f$ is the applied frequency to the signal of $1 \mathrm{~V}, \tau$ is the mean relaxation time, and $\alpha$ is the spreading factor of the actual relaxation times about the mean value.

The pioneering work of Cole-Cole and the standard procedure was adopted in this work to fit the data and to obtain $\alpha$ value for CFMO materials. ${ }^{43}$ However, we have considered the real part of dielectric constant instead of the complex part of dielectric constant, we plotted the graph of $\log _{\mathrm{e}}\left[\left(\varepsilon_{0}^{\prime}-\varepsilon^{\prime}\right) /\left(\varepsilon^{\prime}-\varepsilon_{\infty}^{\prime}\right)\right]$ versus $\log _{\mathrm{e}}(f)$ for all the compositions $(x)$. The data obtained are shown in Figure 9. It can be seen that the data obtained for CFMO compounds yield straight lines for various $x$ values. Slope of the linear plot for each composition provides the value of $(1-\alpha)$ from which $\alpha$ is determined. Interestingly, " $\alpha$ " values estimated by this procedure not only matches well with those reported in the literature for the similar materials but also reasonably produce the expected values of the relaxation time $(\tau)$. The calculated values of spreading factor $(\alpha)$ and the relaxation time $(\tau)$ are listed in Table II for all the compositions. Thus, with these values of $\tau$ and $\alpha$, the experimentally measured room temperature frequency dependence of $\varepsilon^{\prime}$ is fitted with the model, Eq. (4), and is as shown in Fig. 10. The experimental data is in good agreement with the calculated data indicating the validity of modified Debye's function with 

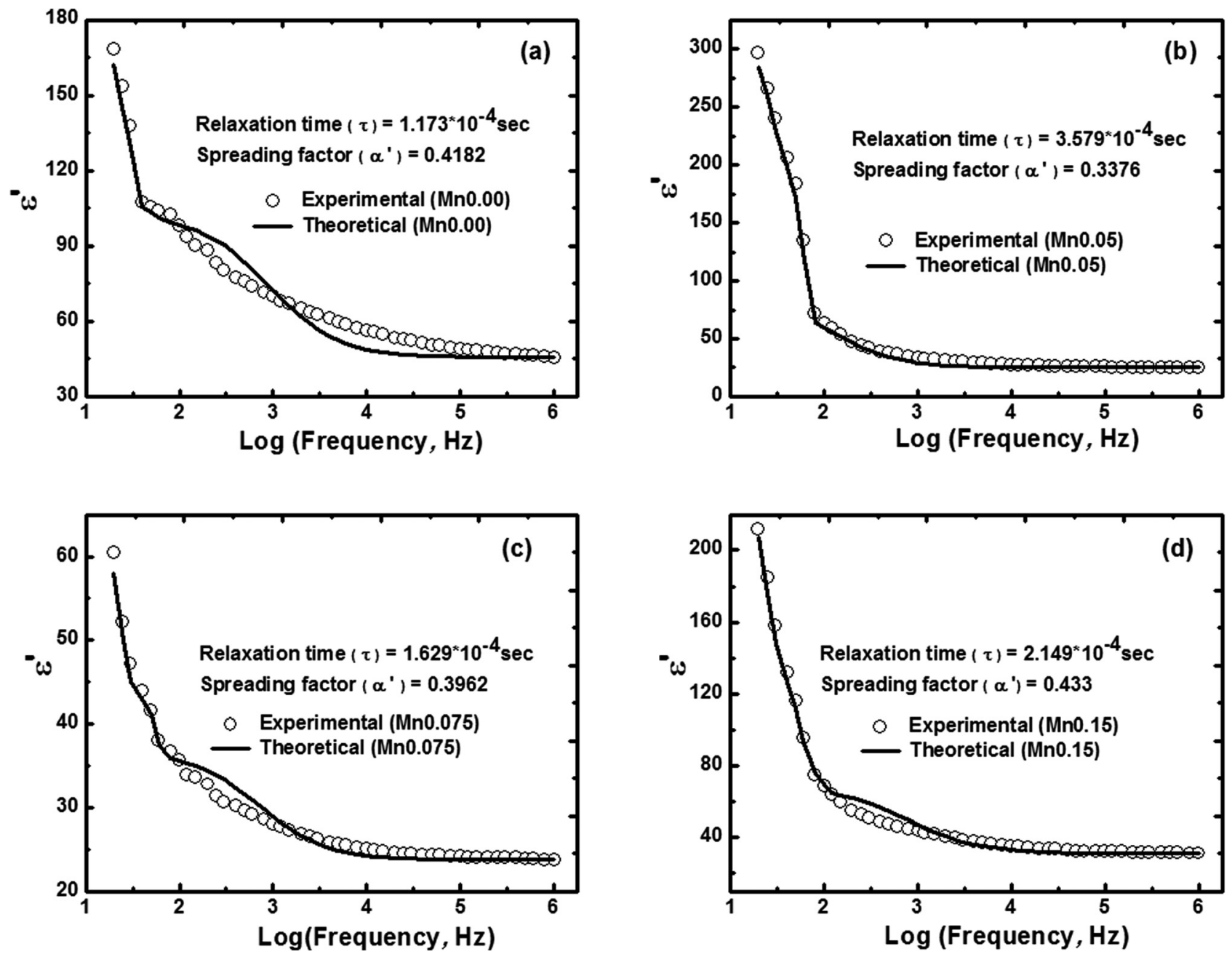

FIG. 10. Room temperature frequency dependent real part of dielectric constant $\left(\varepsilon^{\prime}\right)$ for $\mathrm{CoFe}_{2-x} \mathrm{Mn}_{x} \mathrm{O}_{4}$ ferrites. The data are fitted with the modified Debye model taking the spreading factor $(\alpha)$ determined from Fig. 9 into account. The experimental data and calculation agree well.

the possibility of more than one ion contributing to the relaxation process.

\section{CONCLUSIONS}

The structural, magnetic, and dielectric properties of $\mathrm{CoFe}_{2-\mathrm{x}} \mathrm{Mn}_{\mathrm{x}} \mathrm{O}_{4}(x=0.00-0.15)$ ferrites were evaluated. All of these $\mathrm{CoFe}_{2-\mathrm{x}} \mathrm{Mn}_{\mathrm{x}} \mathrm{O}_{4} \quad(x=0.00-0.15)$ ferrites exhibit inverse cubic spinel structure with long range ferromagnetic ordering. Experimental data coupled with theoretical analysis of the structure and magnetism data for CFMO clearly indicate that Mn ions initially (5\%) compete for occupancy of the $\mathrm{Co}^{2+}$ and $\mathrm{Fe}^{3+}$ ions followed by complete substitution for $\mathrm{Fe}^{3+}$ ions alone for higher $\mathrm{Mn}$ concentration (>5\%). The cubic anisotropy constant $\left(K_{1}(T)\right)$ and saturation magnetization $\left(M_{s}(T)\right)$ determined by the "law of approach" to saturation indicates that these parameters decrease with increasing temperature. Data analysis revealed $M_{s}$ - T functional relationship obeys the Bloch's law in $\mathrm{CoFe}_{2-\mathrm{x}} \mathrm{Mn}_{\mathrm{x}} \mathrm{O}_{4}(x=0.00-0.15)$ ferrites. Correlated to the structure and magnetism, the dielectric constant dispersion of CFMO in the frequency range of $20 \mathrm{~Hz}-1 \mathrm{MHz}$ fits to the modified Debye's function with multiple ions from CFMO contributing to the relaxation. The relaxation time and spread factor derived from modeling the experimental data are $\sim 10^{-4} \mathrm{~s}$ and $\sim 0.35$ ( \pm 0.05$)$, respectively, which are expected for CFO-based materials.

${ }^{1}$ R. Nongjai, S. Khan, K. Asokan, H. Ahmed, and I. Khan, J. Appl. Phys. 112, $084321(2012)$

${ }^{2}$ K. Maaz, M. Usman, S. Karim, A. Mumtaz, S. K. Hasanain, and M. F. Bertino, J. Appl. Phys. 105, 113917 (2009).

${ }^{3}$ N. Ponpandian, P. Balaya, and A. Narayanasamy, J. Phys.: Condens. Matter 14, 3221-3237 (2002).

${ }^{4}$ R. Valenzuela, Phys. Res. Int. 2012, 9, article no. 591839.

${ }^{5}$ B. Zhou, Y.-W. Zhang, Y.-J. Yu, C.-S. Liao, C.-H. Yan, L.-Y. Chen, and S.-Y. Wang, Phys. Rev. B 68, 024426 (2003).

${ }^{6}$ B. H. Liu, J. Ding, Z. L. Dong, C. B. Boothroyd, J. H. Yin, and J. B. Yi, Phys. Rev. B 74, 184427 (2006).

${ }^{7}$ R. M. Bozorth, E. F. Tilden, and A. J. Williums, Phys. Rev. 99, 1788 (1955).

${ }^{8}$ G. A. Petitt and D. W. Forester, Phys. Rev. B 4, 3912-3923 (1971); H. Shenker, Phys. Rev. 107, 1246-1249 (1957).

${ }^{9}$ J. A. Paulsen, A. P. Ring, C. C. H. Lo, J. E. Snyderb, and D. C. Jiles, J. Appl. Phys. 97, 044502 (2005).

${ }^{10} \mathrm{~S}$. Chikazumi, Physics of Ferromagnetism, 2nd ed. (Oxford University Press, Oxford, 1997).

${ }^{11}$ J. Smith and H. P. J. Wijn, Ferrites (Philips Technical Library, Eindhoven, Holland, 1965).

${ }^{12}$ K. Kamala Bharathi and C. V. Ramana, J. Mater. Res. 26, 584 (2011).

${ }^{13}$ K. Kamala Bharathi, R. J. Tackett, C. E. Botez, and C. V. Ramana, J. Appl. Phys. 109, 07A510 (2011). 
${ }^{14}$ K. Kamala Bharathi, G. Markandeyulu, and C. V. Ramana, J. Phys. Chem. C 115, 554 (2011).

${ }^{15}$ I. C. Nlebedim, N. Ranvah, Y. Melikhov, P. I. Williams, J. E. Snyder, A. J. Moses, and D. C. Jiles, J. Appl. Phys. 107, 09A936 (2010).

${ }^{16}$ C. C. H. Lo, J. Appl. Phys. 107, 09E706 (2010).

${ }^{17}$ K. Krieble, M. Devlin, S. J. Lee, S. T. Aldini, and J. E. Snyder, J. Appl. Phys. 103, 07E508 (2008).

${ }^{18}$ N. Ranvah, Y. Melikhov, D. C. Jiles, J. E. Snyder, A. J. Moses, P. I. Williams, and S. H. Song, J. Appl. Phys. 103, $07 E 506$ (2008).

${ }^{19}$ J. Lee, C. C. H. Lo, P. N. Matlage, S. H. Song, Y. Melikhov, J. E. Snyder, and D. C. Jiles, J. Appl. Phys. 102, 073910 (2007).

${ }^{20}$ S. H. Song, C. C. H. Lo, S. J. Lee, S. T. Aldini, J. E. Snyder, and D. C. Jiles, J. Appl. Phys. 101, 09C517 (2007).

${ }^{21}$ Y. Melikhov, J. E. Snyder, C. C. H. Lo, P. N. Patlage, S. H. Song, K. W. Dennis, and D. C. Jiles, IEEE Trans. Magn. 42, 2861 (2006).

${ }^{22}$ F. Cheng, C. Liao, J. Kuang, Z. Xu, C. Yan, L. Chen, H. Zhao, and Z. Liu, J. Appl. Phys. 85, 2782 (1999).

${ }^{23}$ G.-L. Sun, J.-B. Li, J.-J. Sun, and X.-Z. Yang, J. Magn. Magn. Mater. 281, 173 (2004).

${ }^{24}$ K. Kamala Bharathi, K. Balamurugan, P. N. Santhosh, M. Pattabiraman, and G. Markandeyulu, Phys. Rev. B 77, 172401 (2008).

${ }^{25}$ P. A. Shaikh, R. C. Kambale, A. V. Rao, and Y. D. Kolekar, J. Magn. Magn. Mater. 322, 718 (2010).

${ }^{26}$ R. C. Kambale, P. A. Shaikh, C. H. Bhosale, K. Y. Rajpure, and Y. D. Kolekar, Smart Mater. Struct. 18, 115028 (2009).

${ }^{27}$ S. D. Bhame and P. A. Joy, J. Phys. D: Appl. Phys. 40(11), 3263 (2007).

${ }^{28}$ S. D. Bhame and P. A. Joy, J. Appl. Phys. 100, 113911 (2006).

${ }^{29}$ K. Krieble, T. Schaeffer, J. A. Paulsen, A. P. Ring, C. C. H. Lo, and J. E. Snyder, J. Appl. Phys. 97, 10F101 (2005).
${ }^{30}$ Y. Melikhov, J. E. Snyder, D. C. Jiles, A. P. Ring, J. A. Paulsen, C. C. H. Lo, and K. W. Dennis, J. Appl. Phys. 99, 08R102 (2006).

${ }^{31}$ J. A. Paulsen, A. P. Ring, C. C. H. Lo, J. E. Snyder, and D. C. Jiles, J. Appl. Phys. 97, 044502 (2005).

${ }^{32}$ O. Caltun, H. Chiriac, N. Lupu, I. Dumitru, and B. Rao, J. Optoelectr. Adv. Mater. 9(4), 1158 (2007).

${ }^{33}$ G. D. Rieck and J. J. M. Thijssen, Acta Crystallogr. B 24, 982 (1968).

${ }^{34}$ A. Franco, F. L. A. Machado, and V. S. Zapf, J. Appl. Phys. 110, 053913 (2011).

${ }^{35}$ M. Atif, R. Sato Turtelli, R. Grossinger, and F. Kubel, J. Appl. Phys. 113, 153902 (2013).

${ }^{36}$ H. Kronmüller, D. Goll, I. Kleinschroth, and A. Zern, "Advanced hard and soft magnetic materials," Mater. Res. Soc. Symp. Proc. 577, 303 (1999).

${ }^{37}$ J. C. Maxwell, Electricity and Magnetism (Oxford University Press, London, 1973).

${ }^{38}$ K. W. Wagner, Ann. Phys. 40, 817 (1993).

${ }^{39}$ C. G. Koops, Phys. Rev. 83, 121 (1951).

${ }^{40}$ L. T. Rabinkin and Z. I. Novikova, Ferrites 146 I2V (Acad. Nauk. USSR, Minsk, 1960).

${ }^{41}$ N. Popandian, P. Balaya, and A. Narayanasamy, J. Phys.: Condens. Matter. 14, 3221 (2002).

${ }^{42}$ R. S. Devan, Y. D. Kolekar, and B. K. Chougule, J. Phys.: Condens. Matter. 18, 9809 (2006).

${ }^{43}$ K. S. Cole and R. H. Cole, J. Chem. Phys. 9, 341 (1941).

${ }^{44}$ J. C. Anderson, Dielectrics (Spottiswoode, Ballantyne \& Co Ltd, London and Colchester, 1964).

${ }^{45}$ J. Smith and H. P. J. Wijn, Ferrites (Philips Technical Library, Eindhoven, The Netherlands, 1965). 